\title{
Anterior temporal cortex and semantic memory: Reconciling findings from neuropsychology and functional imaging
}

\author{
TIMOTHY T. ROGERS \\ MRC Cognition and Brain Sciences Unit, Cambridge, England \\ and University of Wisconsin, Madison, Wisconsin \\ JULIA HOCKING and UTA NOPPENEY \\ Functional Imaging Laboratory, London, England \\ ANDREA MECHELLI \\ Functional Imaging Laboratory, London, England \\ and King's College London, London, England \\ MARIA LUISA GORNO-TEMPINI \\ University of California, San Francisco, California \\ KARALYN PATTERSON \\ MRC Cognition and Brain Sciences Unit, Cambridge, England \\ and \\ CATHY J. PRICE \\ Functional Imaging Laboratory, London, England
}

\begin{abstract}
Studies of semantic impairment arising from brain disease suggest that the anterior temporal lobes are critical for semantic abilities in humans; yet activation of these regions is rarely reported in functional imaging studies of healthy controls performing semantic tasks. Here, we combined neuropsychological and PET functional imaging data to show that when healthy subjects identify concepts at a specific level, the regions activated correspond to the site of maximal atrophy in patients with relatively pure semantic impairment. The stimuli were color photographs of common animals or vehicles, and the task was category verification at specific (e.g., robin), intermediate (e.g., bird), or general (e.g., animal) levels. Specific, relative to general, categorization activated the antero-lateral temporal cortices bilaterally, despite matching of these experimental conditions for difficulty. Critically, in patients with atrophy in precisely these areas, the most pronounced deficit was in the retrieval of specific semantic information.
\end{abstract}

Studies of patients with semantic impairment suggest that the most anterior portions of the temporal cortices critically support human conceptual knowledge. The purest documented semantic syndrome, semantic dementia (SD; Hodges, Patterson, Oxbury, \& Funnell, 1992; Snowden, Goulding, \& Neary, 1989), arises from progressive deterioration of the anterior temporal cortex, frequently more pronounced in the left hemisphere, but always involving both (Davies, Graham, Xuereb, Williams, \& Hodges, 2004; Mummery et al., 2000). Patients with SD are impaired on any task requiring knowledge about the meanings of words and objects, including picture naming, word-picture matching, category and property verification (Rogers et al., 2004;

Correspondence concerning this article should be addressed to T. T. Rogers, Department of Psychology, University of Wisconsin, W. J. Brogden Hall, 1202 W. Johnson St., Madison, WI 53702 (e-mail: ttrogers@wisc.edu).
Rogers, Watling, Hodges, \& Patterson, 2005; Snowden et al., 1989; Warrington, 1975), matching pictures or words on the basis of thematic associations (Hodges, Graham, \& Patterson, 1995), sorting words or pictures (Hodges et al., 1992; Rogers et al., 2004), drawing-to-name and delayed copying of drawings of familiar objects (Bozeat et al., 2003; Rogers et al., 2004), sound-picture matching (Bozeat, Lambon Ralph, Patterson, Garrard, \& Hodges, 2000), demonstrating the correct use of objects (Hodges, Bozeat, Lambon Ralph, Patterson, \& Spatt, 2000), object reality decision (Rogers, Lambon Ralph, Hodges, \& Patterson, 2003), and so on (see Patterson \& Hodges, 2000). These deficits are typically observed for all semantic categories (Garrard, Lambon Ralph, \& Hodges, 2002) and are apparent in all modalities of testing but are specific to semantic knowledge; other cognitive faculties are reasonably well preserved in the disorder (Hodges, Garrard, \& Patterson, 1998). The striking consistency of both the cognitive and the neural abnormalities in SD strongly suggests that the 
bilateral anterior temporal cortices are critical for amodal and domain-general aspects of semantic processing (Rogers et al., 2004); that is, they contribute to semantic processing for all kinds of concepts and for all modalities of reception and expression. In line with this view, other brain diseases that can affect the anterior temporal lobes (ATLs), such as Alzheimer's disease and herpes simplex viral encephalitis, also often disrupt semantic memory, although never as selectively as in SD (Hodges \& Patterson, 1995).

Functional neuroimaging has offered a rather startlingly different picture of the neural representation of semantic knowledge, in three respects. First, the majority of research has yielded left-sided, rather than bilateral, cortical activations for semantic tasks (Devlin et al., 2002; Joseph, 2001; Martin \& Chao, 2001; Thompson-Schill, 2003). Second, functional imaging results have indicated that semantic knowledge is encoded in a widely distributed cortical network, with different regions specialized to represent particular kinds of information (Martin \& Chao, 2001; Tranel, Damasio, \& Damasio, 1997), particular categories of object (Caramazza \& Mahon, 2003; Perani et al., 1995), or both (Humphreys \& Forde, 2001; Thompson-Schill, Aguirre, D'Esposito, \& Farah, 1999)_-leading some researchers to suggest that no single region supports semantic abilities for all modalities and categories (e.g., ThompsonSchill, 2003). Third and perhaps most puzzling, although anterior temporal activation has been associated with sentence comprehension (Crinion, Lambon Ralph, Warburton, Howard, \& Wise, 2003; Davis \& Johnsrude, 2003; Mazoyer et al., 1993; Vandenberghe, Nobre, \& Price, 2002), famous face recognition (Gorno-Tempini \& Price, 2001; GornoTempini et al., 1998; Grabowski et al., 2001; Tranel et al., 1997), and a few other semantic tasks (Devlin et al., 2000; Gauthier, Anderson, Tarr, Skudlarski, \& Gore, 1997; Mummery et al., 1999; Ricci et al., 1999; Tyler et al., 2004; Vandenberghe, Price, Wise, Josephs, \& Frackowiak, 1996), the vast majority of functional imaging studies have reported posterior temporal and/or frontal activations for semantic tasks, with no mention of the anterior temporal cortex (for recent literature reviews, see Joseph, 2001; Martin \& Chao, 2001; Thompson-Schill, 2003).

To summarize, neuropsychological research on SD might lead one to conclude that although many other regions undoubtedly contribute, the anterior temporal cortex in both hemispheres is critical for semantic representation and processing across all stimulus modalities and for all types of conceptual knowledge. From functional imaging research on normal adults, in contrast, one might conclude that no single region contributes to semantic memory for all modalities and categories and, further, that the widely distributed network responsible for different aspects of semantic processing is left-lateralized and includes the posterior temporal and frontal cortex, but not the ATL. How is this conundrum to be resolved?

\section{A Convergence Theory of Semantic Processing}

We have previously proposed that the ATL regions affected in SD serve, in the healthy brain, to mediate communication among the modality-specific regions distributed throughout the cortex that encode explicit representations of object attributes (McClelland \& Rogers, 2003; Rogers et al., 2004) — a hypothesis that is supported both by neuroanatomical (Gloor, 1997) and computational (Rogers \& McClelland, 2004) considerations and by the neuropsychological phenomena described previously. When this cross-modal "hub" degrades as a consequence of brain disease, the ability to map between surface forms - for instance, to generate an item's name from its visual image or vice versa-is compromised. On this view, the ATLs critically support semantic task performance for all modalities of reception and expression and all categories of objects, as suggested by the impairments observed in SD. Perhaps less intuitively, this hypothesis also offers a clue regarding the discrepancy in the data from neuropsychology and functional imaging.

The key observation is that in SD, knowledge about properties that individuate a specific concept from its semantic neighbors (e.g., the stripes of a zebra) is always more vulnerable than knowledge about properties shared by related concepts (e.g., the fact that a zebra has four legs). This pattern has been documented in tasks as varied as naming, word-picture matching, drawing, object recognition, coloring, lexical decision, and object use (Papagno \& Capitani, 2001; Patterson et al., 2006; Rogers et al., 2004; Snowden, Neary, \& Mann, 1996; Warrington, 1975). Moreover, this pervasive tendency does not simply reflect the overall difficulty of retrieving more specific, relative to more general, information. For instance, healthy controls are typically faster and more accurate in classifying objects at the basic level (e.g., bird) than at more general levels (Rosch, Mervis, Gray, Johnson, \& Boyes-Braem, 1976), but the reverse is true for patients with SD (Hodges et al., 1995; Rogers, Watling, et al., 2005). Thus, tasks that require objects to be classified with greater precision appear to exert greater demands on the neural system affected in SD, even when they are not more difficult overall. One basis for the discrepancy between neuropsychology and functional imaging, then, may be that functional imaging studies have not tended to use semantic tasks that require very specific classification of the stimulus.

The few functional imaging studies that do report ATL effects appear to be generally consistent with this idea, although the evidence to date is equivocal and other interpretations have been offered. For instance, most of these studies concern recognition or naming of famous or familiar people and/or buildings (H. Damasio, Grabowski, Tranel, \& Hichwa, 1996; Gorno-Tempini \& Price, 2001; Gorno-Tempini et al., 1998; Grabowski et al., 2001; Nakamura et al., 2000; Nakamura et al., 2001; Sugiura et al., 2001) - suggesting to some researchers that the ATLs are dedicated to the representation and processing of lexical or semantic information about unique entities but are not otherwise involved in semantic memory (Tranel et al., 1997). As we and others have previously noted (Gauthier et al., 1997; Gorno-Tempini \& Price, 2001; Gorno-Tempini et al., 1998), however, this pattern may not reflect functional specialization for unique items per se but may, instead, arise from a more general sensitivity within the ATLs to specific 
classification. That is, recognition of unique entities may constitute a form of very specific recognition carried out by the very same processes that support classification of nonunique items into fairly specific semantic categories.

To our knowledge, only two previous functional imaging studies have directly compared different levels of classification for nonunique items. In the first, Gauthier et al. (1997) used fMRI to investigate temporal lobe activations in subjects classifying line drawings of common objects at the subordinate (e.g., flamingo) or basic (e.g., bird) level. The authors found no selective anterior temporal activation in the direct contrast of specific to basic-level categorization; however, such an effect was observed bilaterally after activations from a second, purely verbal semantic task were subtracted out. Although suggestive, the results are somewhat difficult to interpret, given that the effect was apparent only in a double subtraction with another semantic task. Furthermore, the subjects were slower and less accurate to respond in the specific, relative to the general, condition; hence, the impact of the specificity manipulation was potentially confounded with task difficulty.

A second study compared basic and general classification (Tyler et al., 2004). Subjects silently named photographs of objects at the basic level (e.g., dog) or at a very general level (e.g., living thing). Using event-related fMRI, the authors observed activation on the medial surface of the left ATL within the perirhinal cortex. This contrasts with the lateral anterior temporal activation observed for subordinate, relative to basic, categorization by Gauthier et al. (1997). Furthermore, although patients with SD have dramatically reduced volume in both medial and lateral anterior temporal regions (Davies et al., 2004; Mummery et al., 2000), patients with antero-medial damage sparing the lateral cortex do not show the same all-encompassing semantic impairments observed in SD (Levy, Bayley, \& Squire, 2004; Moss, Rodd, Stamatakis, Bright, \& Tyler, 2005).

In sum, there appears to be little evidence from functional imaging to refute the view of semantic processing suggested by behavioral impairments in SD-that the ATLs contribute to semantic memory for all kinds of objects and are most strongly taxed by tasks that require specific classification of the stimulus. Nor, however, is there strong evidence from imaging to support this view, and a consideration of the behavioral and imaging literatures together raises several open empirical questions about the role of the anterior temporal cortex in semantic memory: (1) Are the ATLs activated by specific, relative to more general, semantic classification tasks when overall difficulty is controlled? (2) If so, are the areas of activation in healthy controls situated medially, laterally, or both? (3) Are patterns of anterior temporal activation in semantic processing observed bilaterally, as the behavioral profile of SD would suggest, or are they left-lateralized, as most functional imaging studies show? (4) Are patterns of ATL activation observed across all domains of conceptual knowledge, as suggested by $\mathrm{SD}$, or are they selective to some categories, as they appear to be in other parts of the brain from some functional imaging studies (Chao,
Haxby, \& Martin, 1999)? (5) Are the ATL regions activated by unique-item identification different from regions activated by specific classification (Tranel et al., 1997) or the same (Gauthier et al., 1997)?

In the present work, we addressed these questions with positron emission tomography (PET), since it is notoriously difficult to get a good signal from the ATLs in fMRI, especially at higher field strengths (Devlin et al., 2000). We used a category verification paradigm in which healthy subjects categorized pictures of animals and artifacts at specific, intermediate, and general levels (see Figure 1). On each trial, the subjects decided whether a color photograph matched a general name (e.g., animal or vehicle), an intermediate name (e.g., bird or car), or a specific name (e.g., robin or Volkswagen). A baseline task was also included to identify activation common to all the levels of categorization. Prior to the PET study, stimulus items were normed in a behavioral pilot study, to ensure that the subjects were equally fast and accurate at verifying category membership (1) at the most general and most specific levels and (2) for animals and vehicles at both intermediate and specific levels (see the Method section for details).

To permit rigorous tests of the questions posed above, we first identified regions of interest (ROIs) in the ATLs on the basis of gray matter loss in a group of 6 patients with SD. We then investigated functional activation evoked in these regions (in normal individuals) by specific, relative to more general, semantic categorization, for both animals and vehicles. Finally, to assess whether specific classification engaged the same regions as unique-item identification, we will report functional imaging data from a previous study of naming unique faces versus nonunique items (Gorno-Tempini, Cipolotti, \& Price, 2000) that were collected on the same scanner and analyzed with the same procedures as in the present task.

\section{METHOD}

\section{Patients}

The site of maximal atrophy in SD was determined on the basis of structural T1-weighted MRI anatomical brain images from the 6 patients previously reported by Mummery et al. (2000) and 60 neurologically normal control subjects. All the images were acquired using a 2T Siemens Vision system with identical protocols for each subject. Using standard procedures in SPM2 (Wellcome Department of Imaging Neuroscience, London, www.fil.ion.ucl.ac.uk/spm), all 66 images were spatially normalized, segmented into gray and white matter, and smoothed with a Gaussian kernel of $12 \mathrm{~mm}^{3}$. To identify the gray matter loss in each individual patient, his or her image was compared with those of 10 age- and sex-matched neurologically normal controls. The six differences between the patients and their individual control groups were then averaged to yield a T map of the mean amount of gray matter reduction across patients (Gitelman, Ashburner, Friston, Tyler, \& Price, 2001).

\section{Healthy Subjects}

Twelve male subjects (age, 19-39 years; mean age, 25) participated in the functional imaging study. All were right-handed native English speakers, were free from any history of neurological disease or mental illness, and were not on any medication. The study was approved by the local hospital ethics committee and the Administration of Radioactive Substances Advisory Committee (ARSAC, 


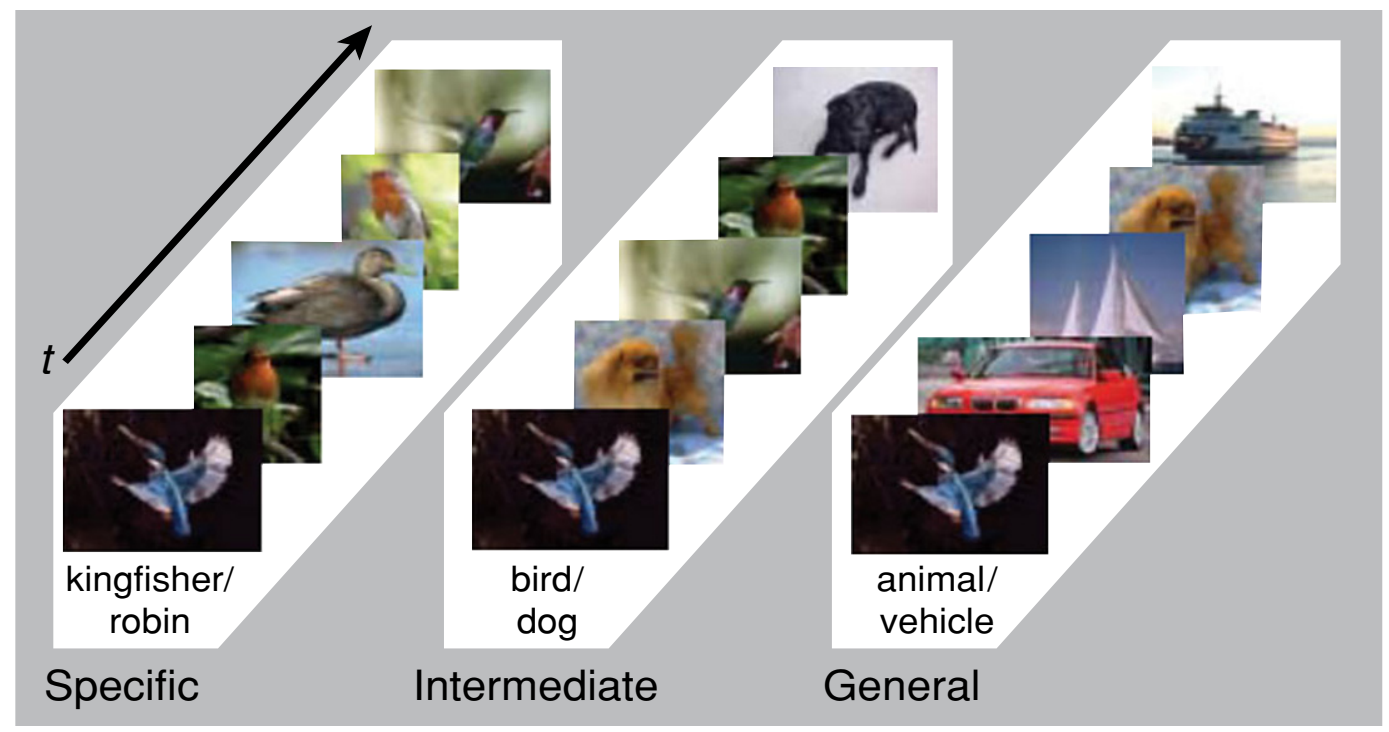

Figure 1. Some examples of the stimuli used and a schematic of the block design. On each trial, a word was viewed, followed by a picture, and the subjects were asked to indicate whether the picture matched the word.

U.K.). All the subjects in the imaging experiment gave written informed consent prior to receiving a PET scanning session consisting of 12 measurements. The behavioral task was also piloted outside the scanner on a separate set of 12 male subjects, also ranging in age from 19 to 39 years, selected from the volunteer subject pool at the MRC Cognition and Brain Sciences Unit in Cambridge.

\section{Stimuli}

The stimuli consisted of 48 color photographs of real animals and vehicles, including the following: robins, kingfishers, and other birds; labradors, Pekinese, and other dogs; BMWs, Morris Travellers (a well-known automobile in the U.K.), and other cars; and ferries, yachts, and other boats. Target items for the specific categories were successfully named at the specific level by university undergraduates with greater than $72 \%$ agreement.

\section{Task and Design}

In each trial, the subjects viewed a category label, followed by a color photograph, and were asked to indicate by buttonpress whether the photograph matched the word. Category labels could be specific names (e.g., labrador, BMW), intermediate names (e.g., dog, car), or general names (e.g., animal, vehicle). Over subjects, exactly the same stimuli were observed in all three experimental conditions. For specific trials, distractors (i.e., trials that should yield a no response) were from the same semantic category as the probe word (e.g., for labrador, the distractor was a different breed of dog). For intermediate trials, the distractors were from a different category in the same superordinate domain (e.g., for $d o g$, the distractor was a different kind of animal); and for general trials, the distractors were from the contrasting semantic domain (e.g., for animal, the distractor was a vehicle).

Trials were blocked in a design manipulating semantic domain (animal or vehicle) and level of specificity (general, intermediate, or specific). Each 16-trial block (one PET scan) included two different category labels at the same level of specificity, ordered at random. For instance, in one specific block, the subjects viewed the probes labrador and Pekingese, each occurring eight times in random order and followed by a matching or nonmatching dog picture. In a separate intermediate block the probes $d o g$ and bird appeared eight times apiece in random order, and in a third general block, the probes animal and vehicle occurred eight times each.
The 48 items were arranged into 4 different blocks in each specificity condition, yielding 12 experimental blocks total, with the same 48 items appearing in all the task conditions. Three baseline blocks were also constructed, in which the subjects viewed the word left or right, followed by a scrambled photograph, and pressed the left or right response button accordingly. The 12 experimental and 3 baseline blocks yielded a total of 15 scans; however, due to the limitations of PET, each subject could complete, at most, 12 scans. Scans were, therefore, distributed across subjects as follows: Each subject completed all 4 specific blocks, 2 of the 4 intermediate blocks, and 3 of the 4 general blocks, with the assignment of intermediate- and general-level blocks counterbalanced across subjects. This design yielded 9 experimental scans per subject, with the 48 photographs viewed an equivalent number of times in each condition across subjects. All the subjects also completed all 3 baseline scans, so that all the subjects received 12 scans and, across subjects, the same set of photographs was viewed in all three task conditions. The order of the 12 scans was determined randomly for each subject.

Finally, to assess the relative speed and accuracy with which the subjects could classify stimuli at specific, intermediate, and general levels, the task was first piloted outside of the scanner. Although all the subjects in the PET study were encouraged to respond quickly and accurately, requirements of PET can elicit long response times (RTs). Specifically, it is necessary to control the duration and timing of exposure to stimuli during the imaging sessions, so that observed differences in functional activation cannot be attributed to these factors. Consequently, the stimuli appeared on-screen for a constant duration, regardless of a subject's response, and there was a fixed, relatively long intertrial interval (2.5-sec duration from the start of one trial to the start of the next). These conditions can lead subjects to respond relatively slowly and to fall into a "rhythm" of responding locked to the timing of the trials, so that behavioral data collected from the scanning session may not accurately reflect the relative difficulty of the various conditions. The behavioral pilot, therefore, differed from the scanning task in two respects. First, whereas in the imaging session the picture remained on-screen for a fixed duration, in the behavioral pilot it disappeared as soon as a response was detected, at which point the trial ended. Second, the intertrial interval was much shorter $(500 \mathrm{msec}$ from the response to the beginning of the next trial). We anticipated that these minor changes to the 
procedure would encourage the subjects to respond more quickly and, thus, would yield more reliable data concerning the relative difficulty of the task conditions.

\section{PET Scanning}

The 12 PET scans were obtained using a Siemens/CTI (Model 962) PET scanner (Knoxville, TN). Each scan involved a 20 -sec intravenous bolus of $\mathrm{H} 215 \mathrm{O}$ at a concentration of $55 \mathrm{Mbq} / \mathrm{ml}$ and a flow rate of $10 \mathrm{ml} / \mathrm{min}$ through a forearm cannula. For each subject, a T1-weighted structural magnetic resonance image was also obtained with a 2T Magnetom Vision scanner (Siemens, Erlangen, Germany).

\section{Data Analysis}

The PET data were analyzed with statistical parametric mapping (SPM99, Wellcome Department of Imaging Neuroscience, London, www.fil.ion.ucl.ac.uk/spm) implemented in MATLAB (MathWorks Inc., Natick, MA) using standardized procedures (Friston et al., 1995). The mean image created by the realignment procedure was used to determine the parameters for transforming the images onto the Montreal Neurological Institute average brain. These parameters were then applied to the functional images (Ashburner \& Friston, 1997), and the image was resampled into isotropic $2-\mathrm{mm}^{3}$ voxels. Finally, each image was smoothed with a $16 \mathrm{~mm}$ at full-width half-maximum Gaussian filter. The SPM software was then used to compute multiple linear regression analyses. Condition effects were estimated according to the general linear model at each voxel. To test hypotheses about regionally specific condition effects, these estimates were compared using linear contrasts (balanced within subjects). The resulting set of voxel values for each contrast is an SPM of the $t$ statistic, which was then converted to a $Z$ statistic. The significance level was corrected for multiple comparisons $(Z>$ $4.5, p<.05$, corrected), except in our ROIs, where we lowered the threshold to $p<.001$, uncorrected $(Z>3.09)$. There were three contrasts of interest: (1) all categorization conditions relative to baseline, (2) specific categorizations only relative to baseline, and (3) specific categorization relative to general and intermediate categorization. Other results from this experimental session focusing on the posterior fusiform cortex have previously been reported in Rogers, Hocking, Mechelli, Patterson, and Price (2005).

\section{RESULTS}

\section{Behavioral Data}

Repeated measures ANOVAs of the behavioral pilot data showed that the subjects were faster to respond in the intermediate-level condition [speed: $F(2,22)=8.3$, $p<.002]$ but that neither speed nor accuracy differed reliably for classification at the general and specific levels (mean RTs: specific $=749$, basic $=701$, general $=783$; $p=.18$ for two-tailed contrast of specific to general RT). There were no reliable differences across conditions in error rates $[$ specific $=.03$, basic $=.02$, general $=.04$; $F(2,22)=0.91, p=$ n.s.]. Pilot subjects were also equally fast and accurate to verify category membership for animals and vehicles $[F(1,11)=3.38, p=$ n.s., for speed; $F(1,11)=0.31, p=$ n.s., for accuracy] in both intermediate and specific conditions, with no reliable interaction between semantic category and task condition $[F(1,11)=$ $0.24, p=$ n.s., for speed; $F(1,11)=0.25, p=$ n.s., for accuracy]. These results have previously been reported in Rogers, Hocking, et al. (2005).

Analysis of the behavioral data from the PET sessions showed that RTs from the scanning sessions were more than double those in the behavioral pilot (grand mean from pilot: $743 \mathrm{msec}$; grand mean from scanning session: $1,625 \mathrm{msec} ; p<.001$, two-tailed $t$ test). Mean RTs for correct trials were numerically shorter for the intermediate condition $(1,601 \mathrm{msec})$ than for the general $(1,624 \mathrm{msec})$ or specific $(1,741 \mathrm{msec})$ conditions, and a within-subjects ANOVA showed reliable differences in RT across conditions $[F(2,20)=7.0, p<.005]$. Post hoc contrasts revealed that the responses in the specific condition were reliably slower than those in both the intermediate $(p<$ $.005)$ and the general $(p<.03)$ conditions, with no significant difference between the intermediate and the general conditions ( $p=$ n.s.). The error data showed the same pattern: The subjects made significantly more errors for specific, relative to basic $(p<.007)$ and general $(p<.001)$, categorization. A within-subjects ANOVA investigating the effects of semantic domain (animals or vehicles) on RTs at intermediate and specific levels showed no reliable effect of domain [for RT, $F(1,10)=0.02, p=$ n.s.; for accuracy, $F(1,10)=0.05, p=$ n.s.] and no reliable interaction between domain and level [for RT, $F(1,10)=0.29$, $p=$ n.s.; for accuracy, $F(1,10)=1.1, p=$ n.s.].

In summary, behavioral data from the scanner yielded much longer RTs and a somewhat different pattern of results across conditions than did the behavioral pilot. As was previously indicated, the experimental procedure in the pilot experiment encouraged the subjects to respond as quickly and accurately as possible, whereas the requirements of PET likely led to reduced pressure to respond quickly. We therefore interpret the behavioral data from the pilot experiment as providing a more accurate estimate of the relative difficulty of the three conditions. These results suggest that, when subjects performing this task are required to respond as quickly as possible, (1) RTs and accuracy are matched for specific and general conditions, (2) RTs are shorter at the intermediate level, with an equivalent degree of accuracy, and (3) there is no difference in speed or accuracy for animals versus vehicles in either the intermediate or the specific condition.

\section{PET Results}

The peak coordinates for maximum gray matter atrophy in the SD patients were identified at $[-44,14,-27]$ (see Figure 2). A volume with a radius of $8 \mathrm{~mm}$ around this peak and the corresponding volume in the right temporal cortex defined the ROIs for the imaging analysis.

The comparison of all the semantic categorization scans with the baseline scans yielded significant areas of activation $(p<.05$, corrected for multiple comparisons across the entire brain) in the posterior fusiform cortex and in the occipito-temporal cortex bilaterally (see Table 1) - a pattern that has been reported in a great many studies of semantic task performance (Joseph, 2001) - but no significant activation in the ROIs ( $p>.05$, uncorrected). From this simple contrast, it might seem that the anterior temporal cortex does not contribute to the categorization task. When the general and intermediate conditions were excluded, however, activation in the anterior temporal regions was identified in the whole-brain analysis for spe- 
A
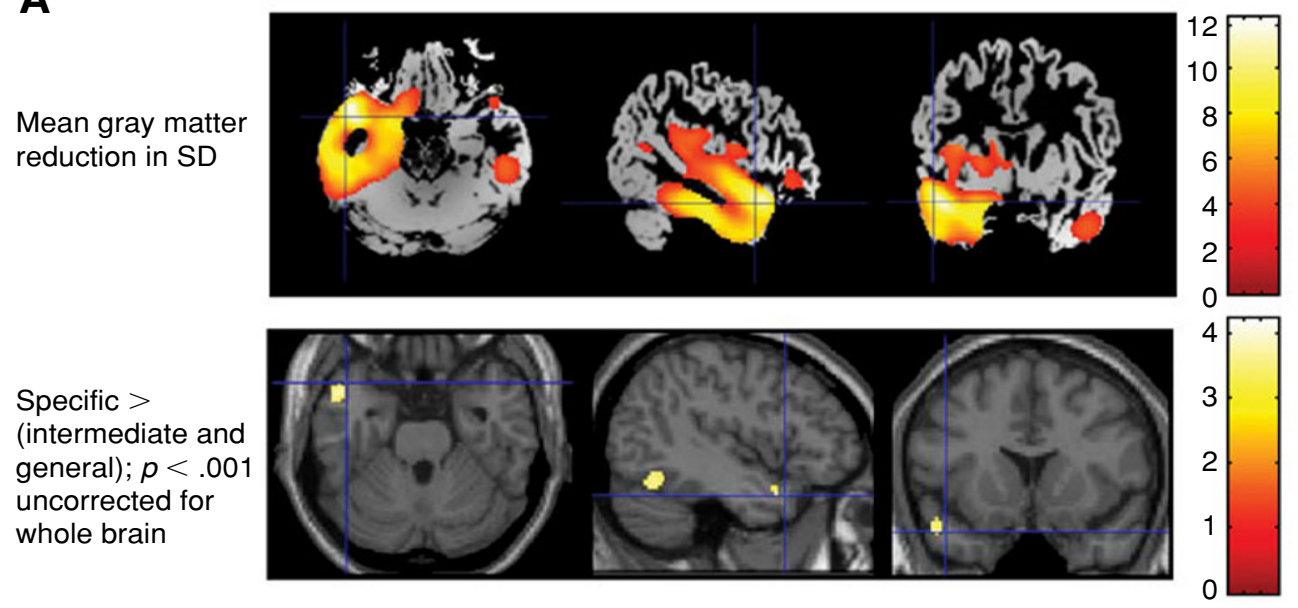

$\mathbf{B}$

Left: $[-\mathbf{5 0}, \mathbf{8},-\mathbf{2 2}]$

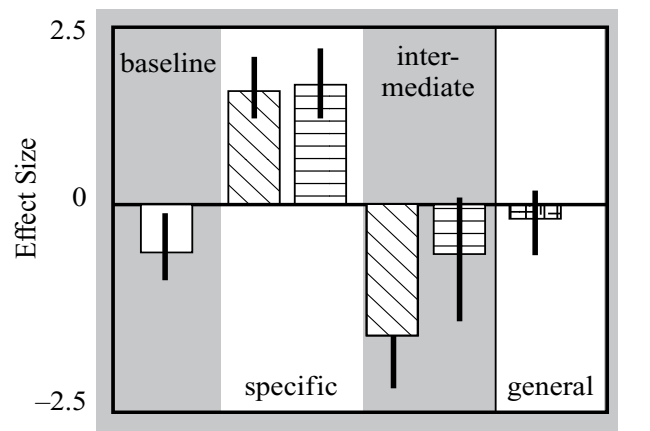

MAnimals $\rightleftharpoons$ Vehicles Intermixed
Right: $[50,8,-22]$

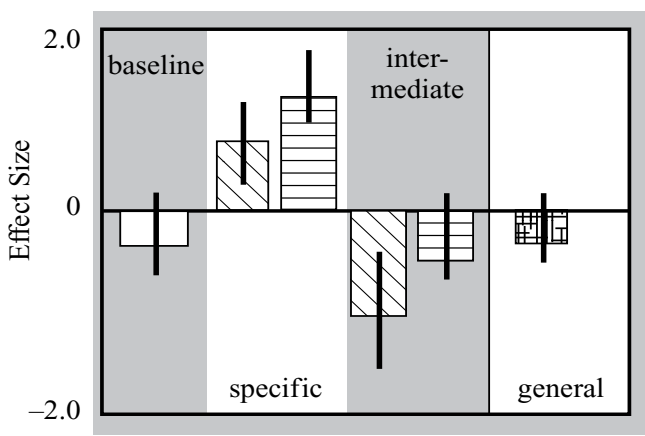

Figure 2. Localization of atrophy and activation. (A) Top row: Mean gray matter reduction for 6 patients with semantic dementia (SD). The peak was identified at $[-44,14,-27]$; a volume of $8 \mathrm{~mm}$ around this peak and the corresponding region in the right hemisphere were defined as regions of interest for the PET analysis. The crosshairs indicate the peak of activation from the functional imaging study contrasting specific with intermediate and general categorization. Bottom row: Wholebrain activation for healthy controls significant at $p<.001$, uncorrected, contrasting the specific with the intermediate and general categorization conditions. A corresponding peak in the right hemisphere did not surmount the whole-brain threshold but fell within the region of interest and was statistically reliable with small-volume correction. The crosshairs indicate the peak of maximal atrophy from the volumetric analysis of SD data. (B) Mean-centered effect sizes (percentage of regional blood flow change) at the left and right peaks for the six different stimulus conditions in our experiment: specific categorization for animals and for vehicles, intermediate categorization for animals and vehicles, general categorization, and the baseline task. The effect was observed for both animal and vehicle scans.

cific categorization, relative to baseline, in both the left $[-54,6,-26](Z=3.6)$ and the right $[48,22,-28](Z=$ 3.1) hemispheres.

The selectivity of this response was confirmed by direct comparison of the specific with general and intermediate categorization, with the left peak at $[-50,8,-22](Z=$ $3.9)$ and the right peak at $[50,8,-22](Z=3.0)$. These activations fall within the area of atrophy in the SD patients, with peak activation in the left corresponding almost exactly to the maximal atrophy in the patients (see Figure 2, panel A). Within the ROI (8-mm radius) around [ -44 , $14,-27]$ and the corresponding region in the right hemisphere $[+44,14,-27]$, activation for the specific $>$ the general and intermediate conditions was significant after correction for multiple comparisons in both hemispheres (left, $p<.001$; right, $p<.03$ ). This effect of specificity was observed for both animal and vehicle categories; there was no main effect of semantic category and no interaction between category and domain (see Figure 2, panel B). No effect of specificity was observed in the medial ATL regions previously investigated in a naming paradigm by Tyler et al. (2004), even using small-volume correction $(p>.05)$.

To verify that the effect of specificity did not solely reflect differences in activation between the specific and the intermediate conditions, we also contrasted specific and 
Table 1

Peak Coordinates From Contrast of All Semantic Tasks With the Baseline Condition



general categorization conditions, which were equated for speed and accuracy in the behavioral pilot. The peak of this contrast was even closer to the peak of atrophy from the patient data (left, $[-44,12,-24], Z=3.0$; right, [48, $10,-24], Z=2.0)$. This effect survived correction for multiple comparisons within the left ROI $(p<.02)$.

To investigate whether there are other cortical regions within the temporal lobes that respond to specific, intermediate, or general categorization, we contrasted each of these conditions with the baseline task. The results, thresholded at $p<.001$, uncorrected for the whole-brain analysis, are shown in Figure 3. Regions colored green showed significant activation in the general condition, relative to baseline; those colored yellow showed significant activation in the intermediate condition, relative to baseline; and those colored red showed significant activation in the specific condition, relative to baseline. As the figure indicates, all three conditions tended to activate broad and overlapping regions in the occipito-temporal and posterior temporal lobes. The specific condition produced the broadest spread of posterior temporal activation; the general condition produced the next-broadest extent; and the intermediate condition, which generated the shortest RTs, produced the narrowest spread within this region. There was no region that significantly activated for intermediateor general-level classification without also activating for specific-level classification. Thus, although the topographic extent of this posterior activation appears to have been modulated by the specificity of the task, there was no clear topographic relationship between specificity and functional organization within the posterior temporal cortex: that is, the different specificity conditions do not appear to have activated different neuroanatomical sites. The specific task was the only condition to show significant activation in the anterior temporal cortex, relative to baseline.

Finally, to assess whether the same antero-temporal regions also respond to the identification of unique items, such as individual faces, we analyzed the present data together with results from a previous study of picture naming (Gorno-Tempini et al., 2000), which was conducted in the same scanner using the same acquisition and analysis protocols. In this study, subjects were instructed to name photographs of famous faces, objects, animals, and body parts. The authors were interested in identifying categoryspecific patterns of activation and, so, did not directly contrast naming for the unique items (faces) versus the nonunique classes (objects, animals, and body parts). For the present study, we reanalyzed these data to investigate this contrast (using the same whole-brain and ROI analysis procedures described in the Method section). The results are shown in the top row of Figure 4, panel A: Naming of unique faces, relative to nonunique items, activated the ATLs bilaterally, with peaks ( $[-52,+14,-26], Z=3.7$; $[+56,+18,-28], Z=4.0)$ very near to those activated by specific classification of common objects in the present study ([ $\pm 50,8,-22])$.

The bottom row of Figure 4, panel A, shows the conjunction of the specific classification results from the present study and the unique-naming results from GornoTempini et al.'s (2000) study. The peak activation was observed in the same left anterior temporal region $([-52$, $12,-24], Z=5.5, p<.05$, corrected for whole brain).

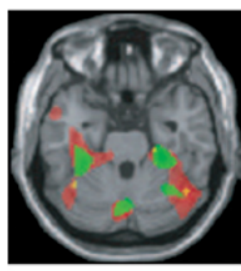

$-26 \mathrm{~mm}$

Intermediate

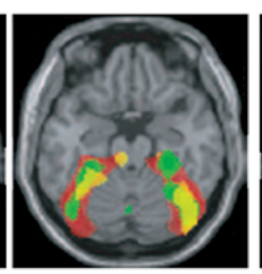

$-24 \mathrm{~mm}$

General

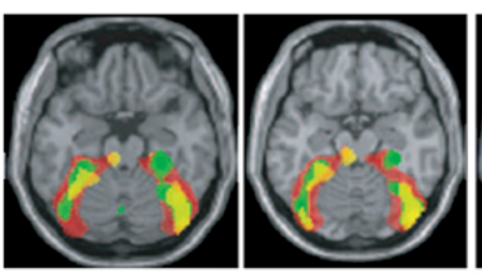

$-20 \mathrm{~mm}$

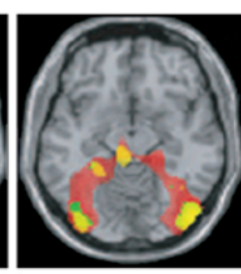

$-12 \mathrm{~mm}$

Specific $p<.001$, uncorrected, no mask

Figure 3. Experimental conditions significantly activated above baseline. Extent of temporal lobe activation was significant at $p<.001$, uncorrected, for the whole brain in the contrast of specific classification with baseline (red), intermediate classification with baseline (yellow), and general classification with baseline (green). 
A

Naming faces $>$ animals, objects, and body parts



Conjunction of (faces $>$ animals, objects, and body parts) with (specific > intermediate and general categorization)

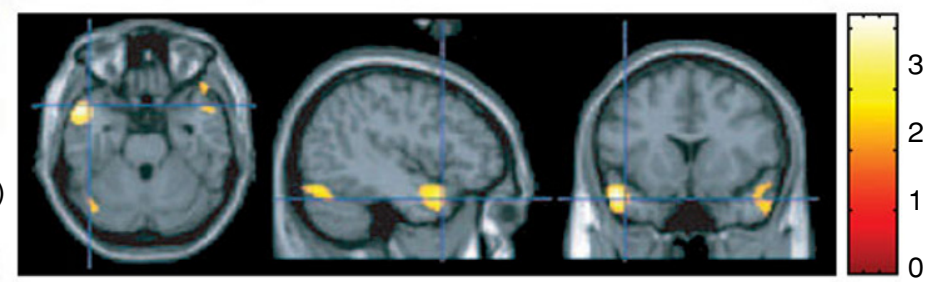

B

Left: $[-46,10,-24]$

Right: $[48,10,-24]$
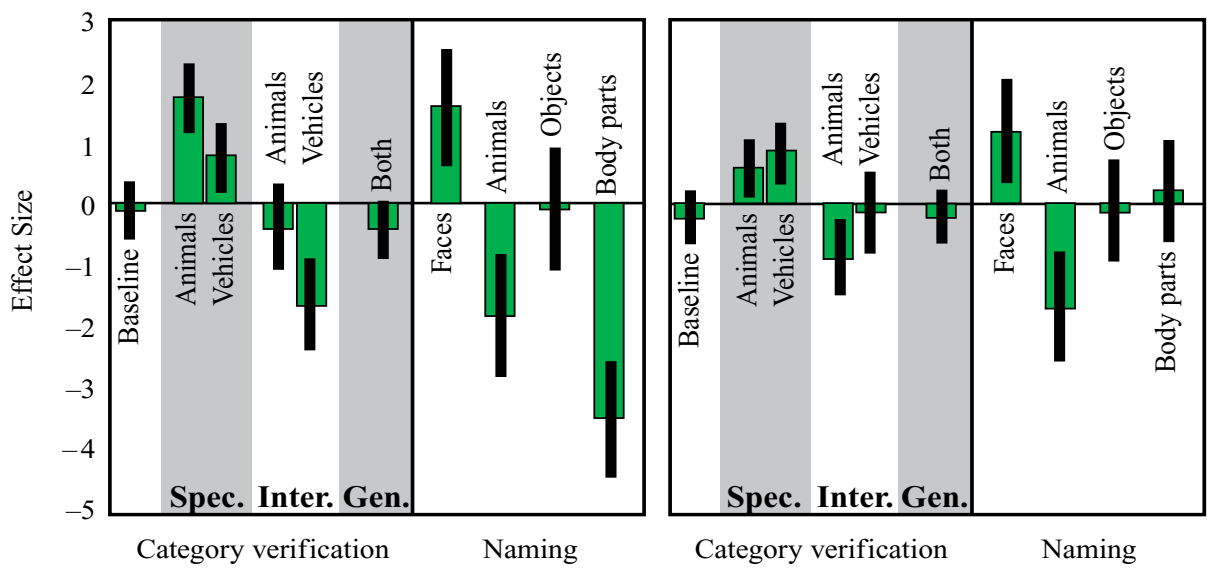

Figure 4. Naming faces versus objects, animals, and body parts. (A) Top: Silent naming of unique faces versus nonunique items (animals, objects, and body parts) from Gorno-Tempini, Cipolotti, and Price (2000). Bottom: Conjunction of (specific $>$ general categorization) from the present study and (naming faces $>$ naming animals, objects, and body parts) from Gorno-Tempini et al. (2000). Note that, in the case of the conjunction, the color scale for the $t$ values relates to the minimal $t$. (B) Mean centered effect sizes (percentages of regional blood flow change) at the peak voxel.

Two corresponding peaks in the right did not reach a corrected level of significance in the whole-brain analysis (Peak 1, [58, 12, -24], $Z=3.5, p=$ n.s.; Peak 2, [50, 14, $-34], Z=3.5, p=$ n.s.) but aligned closely to the righthemisphere ROI. Within these regions, activations in both hemispheres were reliable (left, $[-50,12,-26], \mathrm{Z}=5.4$, $p<.001$, corrected; right, [50, 8, -24], $Z=3.4, p<.01$, corrected). Thus, the same ATL regions most affected in SD appear to be activated in normal individuals both by specific classification of nonunique animals and vehicles and by silent naming of individual people.

Figure 4, panel B, shows the mean-centered effect sizes for the different conditions in the present experiment and the silent-naming experiment in Gorno-Tempini et al. (2000) at the peak voxel of the contrast of the conjunction of specific $>$ general classification for the present study and silent naming of faces, relative to other categories, in Gorno-Tempini et al. (2000) (left, [-46, 10, -24]; right, $[48,10,-24])$. These are very close to the coordinates of peak atrophy in the patients $[-46,14,-27]$. The effect size for silent naming of individual people is approximately equal to that observed for specific categorization of animals and vehicles in the present experiment and is substantially larger than silent naming of any other category of object.

\section{DISCUSSION}

The results show that the ATL regions most affected in SD were significantly activated in healthy individuals classifying pictures of objects and animals at a specific level, relative to more general levels. The activation was bilateral and was equally robust for categorization of 
animals and vehicles, and it appears in the same regions engaged by silent naming of unique faces, relative to nonunique objects, animals, and body parts. Thus, we have shown functional activation in healthy subjects as a result of semantic processing that directly parallels the type of semantic knowledge that is most degraded in SD.

On the basis of the behavioral data from the pilot experiment, the specificity-related activation does not appear to be attributable to the overall difficulty of specific classification; when the subjects were encouraged to respond as quickly and accurately as possible, the most general and most specific conditions were matched for speed and accuracy in responding and, hence, were matched for difficulty in this respect. In the behavioral results from the scanning session itself, however, specific classification elicited significantly longer RTs than did intermediate or general classification, so that we cannot rule out the possibility that difficulty contributed to the observed pattern of activation. Under this interpretation, it is still notable that the activation peak appears in the ATLs, indicating that these regions are, indeed, involved in at least the specific condition of the task.

The results suggest that the previously noted puzzling discrepancy between functional neuroimaging and neuropsychology arises from a confluence of methodological factors that, together, conspire against observing ATL activation in functional imaging. First, most commonly used semantic tasks do not require very precise identification of the stimulus. As is illustrated in Figure 3, anterior temporal activation is not much apparent in association with intermediate- or general-level classification. Second, it is difficult to acquire a good signal in this region with fMRI, the most commonly used technique for these investigations (Devlin et al., 2002; Devlin et al., 2000). In the present work, we used PET because it does not suffer from this susceptibility artifact. Third, to gain statistical power in testing their hypotheses, many investigators limit their analyses to cortical regions of a priori interest, which tend to be regions that are easily activated across different tasks and imaging methods. Areas that are difficult to observe in whole-brain studies are less likely to be used as ROIs in subsequent studies; hence, the statistical power of smallvolume correction is only rarely brought to bear on these areas (see Joseph, 2001). We have addressed this challenge in the present study by using structural imaging data from a patient population, rather than previous functional imaging results, to identify regions of a priori interest.

By addressing these three methodological factors, we show that functional imaging and neuropsychology do, in fact, accord fairly well: Both suggest that left and right anterior temporal regions contribute to semantic processing for living and nonliving things, especially in tasks requiring specific classification.

\section{Interpretation of the Effect}

The specificity effect reported in this article indicates that the antero-lateral temporal cortices are more strongly activated by fairly specific classification of items, relative to more general classification. Perhaps the most clear-cut interpretation of the effect arises from the proposal of Martin and Chao (2001) that there exists a topographic gradient in the infero-temporal cortex, so that more specific concepts are represented more rostrally and more general concepts are represented more caudally. This notion provides an intuitive account of observations from $\mathrm{SD}$, in that pathology in the disease typically spreads backward from the temporal pole, so that the fine-tocoarse deterioration of conceptual knowledge coincides with an anterior-to-posterior dissolution of the temporal cortex. The present data are partially consistent with this view, in that the specific classification task engages more anterior regions, relative to baseline, than do the intermediate and general tasks. The one inconsistency is shown in Figure 3: We found no region between the temporal pole and the posterior temporal cortex that responded significantly above baseline for intermediate and general, but not specific, classification, as the specificity gradient hypothesis would predict.

The present results are also consistent with the theory of normal and degraded semantic representation and processing proposed by Rogers et al. (2004). This theory builds on the fairly widespread view that knowledge about the particular properties of objects is encoded in perceptual and motor representations distributed throughout the cortex - with, for instance, knowledge about the characteristic shapes of objects coded in visual regions responsible for shape perception, knowledge about characteristic patterns of movement stored in regions responsible for motion perception, knowledge about colors represented in regions dedicated to color perception, knowledge about actions in motor regions, and so on (Martin \& Chao, 2001). According to Rogers et al. (2004), these widely distributed perceptual, motor, and language representations do not interact with one another directly. Instead, they interact by means of a representational "hub" in the ATLs - so that, for instance, to make inferences about the visual appearance of an object when given its name, perception of the phonological form of the word first provokes activation of an amodal representation in the ATLs, which then passes activation on to the shape, color, and motion regions that code information about the visual properties of the item denoted by the word. On this view, the ATLs serve to mediate communication among the various perceptual, motor, and language representations that constitute the cortical semantic network. They compute these mappings for all kinds of items and for all modalities of reception and expression.

Rogers et al. (2004) argued that the systematic erosion of detailed semantic information observed in SD arises because the intermediating representations encoded in the ATLs capture the degree of semantic relatedness among known concepts. On this view, closely related items (e.g., a robin and a kingfisher) are represented with quite similar patterns of activity in the ATL, whereas semantically unrelated items (e.g., a robin and a yacht) are represented with dissimilar patterns (Hinton \& Shallice, 1991; Plaut \& Shallice, 1993; Rogers \& McClelland, 2004). In the healthy system, this similarity structure promotes semantic generalization and induction; but when anterior tem- 
poral regions deteriorate in disease, the same principle of semantic organization militates against retrieval of properties idiosyncratic to a specific concept. Because such properties are not shared by closely neighboring concepts, the intermediating representations in the anterior temporal cortex must be specified with great precision in order to generate the correct response elsewhere in the cortical network. As these representations degrade in SD, it becomes increasingly difficult to settle upon precisely the right pattern and, hence, to retrieve detailed semantic information. For more general information, the ATL system can yield the correct response, so long as it finds itself in the right representational ballpark, because all neighboring representations will tend to produce the same correct response in the rest of the network (Rogers \& McClelland, 2004). Rogers et al. (2004) used computer simulations to show that this basic idea provides a straightforward account of a very broad array of findings from the study of SD.

We suggest that the present results are consistent with the theory articulated by Rogers et al. (2004) if one assumes that a given region of the cortex in the semantic network must "work hard" whenever the task requires that different responses be produced to stimuli coded with highly overlapping representations in that region. For instance, when viewing a picture of a bird and trying to decide whether it is a robin, the subject must respond yes for robins and no for kingfishers. If the robin and kingfisher have overlapping representations in the ATLs, this region will be taxed to differentiate them sufficiently to drive the correct response. In contrast, when trying to decide whether a given stimulus is an animal, the system must respond yes to robin, kingfisher, and other animals and no to BMW, Volkswagen, and other vehicles. In this case, representations with a high degree of overlap all lead to the same response, whereas representations with little overlap lead to different responses, so the system can generate the appropriate behavior even if the representations are only weakly specified.

Somewhat surprisingly, we found no region of the cortex that was more strongly activated for intermediate than for specific and general categorization. This result is difficult to interpret, however, since the subjects were fastest to verify category membership at the intermediate level (both in the behavioral pilot and in the scanner). There is, therefore, a confound between level of specificity and speed of response when intermediate categorization is contrasted with specific and general categorization. It is possible, for instance, that intermediate categorization produces a strong signal that persists for a shorter period of time, since the behavioral response is generated more rapidly - so that, integrated over time, no mean difference is observed in the relevant contrasts. To disentangle these influences, it will be useful to obtain estimates of the time course of the metabolic response to each stimulus type in an event-related design.

\section{Relationship to Other Work}

Specificity versus uniqueness. Our interpretation is similar in spirit to those proposed in some previous imaging studies that have reported left anterior temporal acti- vation (Devlin et al., 2002; Gorno-Tempini et al., 2000; Gorno-Tempini et al., 1998; Gorno-Tempini, Wenman, Price, Rudge, \& Cipolotti, 2001; Moore \& Price, 1999; Mummery et al., 1999; Vandenberghe et al., 2002), but it differs from the explanations put forward by others. For instance, Damasio and colleagues (Grabowski et al., 2001; Tranel et al., 1997) have suggested that the temporal poles are especially important for the recognition and representation of unique items, which depend upon knowledge of "intrinsic and relational details that are far more complex than those of a nonunique entity" (Damasio, A.R. \& Damasio, H., 1994, p. 68), whereas the recognition or representation of nonunique classes is accomplished by more posterior regions (Tranel et al., 1997). As we have just noted, however, the regions activated by face naming, relative to object, animal, and body part naming, in previous work by Gorno-Tempini et al. (2000) are nearidentical to the anterior temporal regions activated by specific classification in the present study-suggesting that anterior temporal activation for unique items may reflect the general sensitivity of these regions to specific classification. Consistent with this idea, Gorno-Tempini et al. (1998) found that the temporal poles activated bilaterally for both proper and common name reading, but more strongly for the proper names.

Gauthier et al. (1997) also identified bilateral anterior temporal activation when subjects classified pictures of common objects at the specific level, rather than the basic level, using fMRI; however, this pattern was apparent only once activation from a purely verbal semantic task had been subtracted out - suggesting to the authors that the temporal poles contribute particularly to the visual discrimination of items assigned to specific classes. Like Gauthier et al., we believe that such effects reflect differentiation demands, but we are reluctant to attribute them to specifically perceptual processes. Patients with SD rarely confuse visually similar objects when they are not semantically related, but they frequently confuse visually dissimilar items that are semantically related (e.g., calling a trumpet a piano) - suggesting that their difficulty lies in the discernment of specific semantic, rather than visual, relationships (e.g., Graham, Hodges, \& Patterson, 1994). Such patients are also impaired at purely verbal semantic tasks and in all other modalities of reception and expression tested to date (Patterson et al., 2006). Moreover, other PET studies have shown anterior temporal activation in nonvisual semantic tasks (Devlin et al., 2002; Gorno-Tempini et al., 1998; Nakamura et al., 2001; Scott, Blank, Rosen, \& Wise, 2000). Thus, the imaging and neuropsychology together suggest that ATL representations capture cross-modal semantic (rather than purely visual) similarity structure.

Specificity and sentence processing. A body of work on language processing (Crinion et al., 2003; Marinkovic et al., 2003; Scott et al., 2000; Scott, Leff, \& Wise, 2003; Sharp, Scott, \& Wise, 2004) provides extensive evidence that the left ATL is strongly activated by comprehension of spoken and written language and, especially, by richer narratives, such as short stories, relative to individual sentences or scrambled word sequences. These results offer 
further evidence both that the ATLs critically support semantic knowledge and that they are sensitive to specificity; rich narratives, by definition, delineate concepts with greater specificity than do single words or phrases. Interestingly, ATL activations have been observed in this work with fMRI, suggesting that sentence comprehension produces sufficiently strong signal to overcome the susceptibility artifact that generally makes the region difficult to image with such methods.

Specificity in the lateral ATL versus the perirhinal cortex. As was noted earlier, Tyler et al. (2004) recently reported activation on the medial surface of the left ATL when subjects silently named common objects at the basic level (e.g., monkey), relative to a more general identification (living thing) or to baseline. This result is consistent with the interpretation of lesion studies in nonhuman primates (Bussey, Saksida, \& Murray, 2002) - that is, that the perirhinal cortex encodes complex conjunctions of perceptual features necessary for the assignment of familiar objects to more specific classes. In accord with this hypothesis, at least one recent morphometric study has shown that the extent of perirhinal atrophy correlates with the degree of semantic impairment in SD (Davies et al., 2004). Other recent studies, however, have found that patients with pathology confined to antero-medial temporal lobe regions do not show the same profound and allencompassing semantic impairments as those observed in SD (Levy et al., 2004; Moss et al., 2005) - suggesting that the lateral aspects of the anterior temporal cortex may be particularly important to semantic abilities. The present results are more in accord with the latter hypothesis: The specificity-related functional activation observed in the anterior temporal regions was clearly situated more laterally, and no specificity-related activity was observed in the more medial regions previously investigated by Tyler et al. (2004).

There are at least two possible reasons for the discrepancy between these two sets of results. First, Tyler et al. (2004) used a covert-naming paradigm, whereas we used a category verification paradigm that required subjects to compare a photograph with a previously viewed category name. It is possible that the two tasks engage different anterior temporal regions, perhaps because category verification requires the comparison of two stimulus items, whereas naming does not, or for other reasons unrelated to semantic representation per se. The comparison of the present results with those in Gorno-Tempini et al.'s (2000) study of covert naming do not, however, support this interpretation: Anterior temporal activation for naming specific concepts (faces), relative to basic-level concepts (objects), was observed in exactly the same areas as those activated by specific category verification - suggesting that both tasks engage the same antero-lateral regions when they require specific stimulus classification.

A second - and in our view, more likely - potential explanation for the discrepancy in results is that the size of the response set was not controlled in the naming task used by Tyler et al. (2004). In the superordinate naming condition of that experiment, the subjects were instructed to name each item as either a living thing or a man-made object. In the basic naming condition, the subjects were required to generate a separate unique name for every individual stimulus item. Consequently, the subjects were selecting from only two possible responses in the general condition and from a great many possible responses in the basic condition. The basic $>$ general regions of activation found in this study may, therefore, simply reflect overall task difficulty, rather than an influence of semantic specificity per se. In the category verification paradigm used in the present study, the subjects always viewed only two category labels per block, so that response set size was controlled across levels of specificity.

Lateralization. Most reported anterior temporal effects are left-lateralized, a pattern that has contributed to the common view that the semantic system itself is left-lateralized (e.g., Grabowski et al., 2001). In the present work, significant effects were observed in both hemispheres, but right-hemisphere activation was apparent only with the power of small-volume correction. One hypothesis consistent with these observations is the proposal of a bilaterally distributed anterior temporal semantic system that interacts with a left-lateralized phonological system, so that verbal semantic tasks draw more heavily upon the left anterior temporal cortex (Lambon Ralph, McClelland, Patterson, Galton, \& Hodges, 2001). For instance, SD patients with more left than right atrophy are typically worse at verbal than at nonverbal semantic tasks, whereas patients with more right involvement show comparably poor performance in both (Lambon Ralph et al., 2001). In this sense, our results again mirror the behavioral data from $\mathrm{SD}$, in that they reveal bilateral involvement of the ATLs, but with more robust left-hemisphere activation, in a task requiring verbal comprehension of a category name.

Specificity in the ATL versus the fusiform cortex. Finally, we recently reported a similar effect of specificity from the same experiment in the lateral posterior fusiform cortex bilaterally (Rogers, Hocking, et al., 2005), which differed from the present results in one critical respect: Whereas intermediate-level classification produced a strong category effect in the fusiform cortex, this effect was either greatly attenuated (at $[-46,10,-24]$ and $[48$, $10,-24]$; see Figure 4B) or eliminated (at $[-50,8,-22]$ and $[50,8,-22]$, see Figure 2B) in the ATL regions. If the strength of activation does, indeed, reflect the degree to which similar representations must be differentiated in both regions, this in turn suggests that the representations encoded in the lateral posterior fusiform and the ATLs capture different kinds of similarity relations among the stimulus items. For instance, the fusiform cortex may be computing visual/ structural representations of the items, with birds and dogs coded in overlapping representations by virtue of the many visual properties that they share (eyes, legs, heads, mouths, biological textures, contours, and so on). The same birds and dogs may be represented as somewhat more distinct from one another in the anterior temporal regions, by virtue of the many nonvisual semantic properties that differentiate the items (e.g., birds fly, but dogs walk; most breeds of dog are pets, whereas most species of bird are not; and so on). In 
this case, the fusiform cortex would be more susceptible to a crowding effect when processing animals at an intermediate level than would the anterior temporal regions.

Although somewhat speculative, there is some a posteriori support for this hypothesis. Specifically, Rogers et al. (2004) assessed (1) the degree of overlap in verbal descriptions of 64 items from the Snodgrass and Vanderwart (1980) corpus and (2) the degree of overlap in the visual attributes depicted when subjects were asked to draw the same 64 items. The analysis showed that for the 24 animal items included in the sample, there was more overlap in the visual features produced in the drawings (mean Jaccard's distance of 0.79 for all animal pairs) than in the verbal descriptions (mean Jaccard's distance of 0.88 for all animal pairs; see Rogers et al. 2004, Figure 2) — suggesting that these items tend to have more visual than nonvisual properties in common.

Thus, the differences in patterns of activation between the fusiform and the ATLs may be understood as follows: (1) Both areas respond strongly when the task requires differentiation of items with similar representations; (2) the fusiform cortex codes visual similarity, whereas the anterior temporal cortex encodes "semantic" similarity, which is influenced by knowledge of nonvisual characteristics; and (3) animals from different intermediate categories in our study tend to have more overlap in visual than in semantic representations, so that crowding effects for the intermediate categorization of animals are stronger in the posterior fusiform than in the anterior temporal cortices. In other words, the different patterns of activation indicate that different kinds of similarity structure are encoded by different parts of the cortical semantic network.

\section{Conclusion}

We observed bilateral anterior temporal activation for specific, relative to more general, classification, for both living things and artifacts, in regions closely aligned to those affected in patients with SD who fail at tasks requiring specific concept knowledge. The effect cannot reflect simple task difficulty, because, in contrast to previous studies, specific and general conditions were matched for speed and accuracy in a behavioral study that pressured subjects to respond as quickly and accurately as possible. Moreover, the regions activated were nearly identical to those involved in the naming of unique faces, relative to nonunique items. Imaging and neuropsychology together thus suggest that anterior temporal regions in both hemispheres critically support the retrieval of specific semantic information for all classes of objects.

\section{REFERENCES}

Ashburner, J., \& Friston, K. [J.] (1997). Multimodal image coregistration and partitioning: A unified framework. NeuroImage, 6, 209-217. Bozeat, S., Lambon Ralph, M. A., Graham, K. S., Patterson, K., Wilkin, H., Rowland, J., ET AL. (2003). A duck with four legs: Investigating the structure of conceptual knowledge using picture drawing in semantic dementia. Cognitive Neuropsychology, 20, 27-47.

Bozeat, S., Lambon Ralph, M. A., Patterson, K., Garrard, P., \& Hodges, J. R. (2000). Nonverbal semantic impairment in semantic dementia. Neuropsychologia, 38, 1207-1215.
Bussey, T. J., Saksida, L. M., \& Murray, E. A. (2002). Perirhinal cortex resolves feature ambiguity in complex visual discriminations. European Journal of Neuroscience, 15, 365-374.

Caramazza, A., \& Mahon, B. Z. (2003). The organization of conceptual knowledge: The evidence from category-specific semantic deficits. Trends in Cognitive Sciences, 7, 354-361.

Chao, L. L., HaXвy, J. V., \& Martin, A. (1999). Attribute-based neural substrates in temporal cortex for perceiving and knowing about objects. Nature Neuroscience, 2, 913-919.

Crinion, J. T., Lambon Ralph, M. A., Warburton, E. A., Howard, D., \& WiSE, R. J. S. (2003). Temporal lobe regions engaged during normal speech comprehension. Brain, 126, 1193-1201.

Damasio, A. R., \& Damasio, H. (1994). Cortical systems underlying knowledge retrieval. In C. Koch (Ed.), Large-scale neuronal theories of the brain (pp. 61-74). Cambridge, MA: MIT Press.

Damasio, H., Grabowski, T. J., Tranel, D., \& Hichwa, R. D. (1996). A neural basis for lexical retrieval. Nature, 380, 499-505.

Davies, R. R., Graham, K. S., Xuereb, J. H., Williams, G. B., \& Hodges, J. R. (2004). The human perirhinal cortex and semantic memory. European Journal of Neuroscience, 20, 2441-2446.

DAVIS, M., \& JoHNSRUdE, I. (2003). Hierarchical processing in spoken language comprehension. Journal of Neuroscience, 23, 3423-3431.

Devlin, J. T., Russell, R. P., Davis, M. H., Price, C. J., Moss, H. E., FADILI, M. J., \& TYLER, L. K. (2002). Is there an anatomical basis for category-specificity? Semantic memory studies in PET and fMRI. Neuropsychologia, 40, 54-75.

Devlin, J. T., Russell, R. P., Davis, M. H., Price, C. J., Wilson, J., Moss, H. E., ET AL. (2000). Susceptibility-induced loss of signal: Comparing PET and fMRI on a semantic task. NeuroImage, 11, 589-600.

Friston, K. J., Holmes, A. P., Worsley, K. J., Poline, J. B., Frith, C. D., \& Frackowiak, R. S. J. (1995). Statistical parametric maps in functional imaging: A general linear approach. Human Brain Mapping, 2, 189-210.

Garrard, P., Lambon Ralph, M. A., \& Hodges, J. R. (2002). Semantic dementia: A category-specific paradox. In E. M. Forde \& G. W. Humphreys (Eds.), Category specificity in brain and mind (pp. 149-179). Hove, U.K.: Psychology Press.

Gauthier, I., Anderson, A. W., Tarr, M. J., Skudlarski, P., \& Gore, J. C. (1997). Levels of categorization in visual recognition studied with functional MRI. Current Biology, 7, 645-651.

Gitelman, D. R., Ashburner, J., Friston, K., Tyler, L., \& Price, C. J. (2001). Voxel based mophometry of Herpes simplex encephalitis. NeuroImage, 13, 623-631.

GLOoR, P. (1997). The temporal lobe and limbic system. New York: Oxford University Press.

Gorno-Tempini, M. L., Cipolotti, L., \& Price, C. J. (2000). Category differences in brain activation studies: Where do they come from? Proceedings of the Royal Society of London: Series B, 267, 1253-1258.

Gorno-Tempini, M. L., \& Price, C. J. (2001). Identification of famous faces and buildings: A functional neuroimaging study of semantically unique items. Brain, 124, 2087-2097.

Gorno-Tempini, M. L., Price, C. J., Josephi, O., Vandenberghe, R., Cappa, S. F., Kapur, N., \& Frackowiak, R. S. (1998). The neural systems sustaining face and proper-name processing. Brain, 121, 2103-2118.

Gorno-Tempini, M. [L.], Wenman, R., Price, C. [J.], Rudge, P., \& CipolotTi, L. (2001). Identification without naming: A functional neuroimaging study of an anomic patient. Journal of Neurology, Neurosurgery, \& Psychiatry, 70, 397-400.

Grabowski, T. J., Damasio, H., Tranel, D., Boles Ponto, L. L., Hichwa, R. D., \& Damasio, A. R. (2001). A role for left temporal pole in the retrieval of words for unique entities. Human Brain Mapping, 13, 199-212.

Graham, K. S., Hodges, J. R., \& Patterson, K. (1994). The relationship between comprehension and oral reading in progressive fluent aphasia. Neuropsychologia, 32, 299-316.

Hinton, G. E., \& Shallice, T. (1991). Lesioning an attractor network: Investigations of acquired dyslexia. Psychological Review, 98, 74-95.

Hodges, J. R., Bozeat, S., Lambon Ralph, M. A., Patterson, K., \& SpatT, J. (2000). The role of conceptual knowledge in object use: Evidence from semantic dementia. Brain, 123, 1913-1925.

Hodges, J. R., Garrard, P., \& Patterson, K. (1998). Semantic de- 
mentia and Pick complex. In A. Kertesz \& D. G. Munoz (Eds.), Pick's disease and Pick complex. New York: Wiley-Liss.

Hodges, J. R., Graham, N., \& Patterson, K. (1995). Charting the progression in semantic dementia: Implications for the organisation of semantic memory. Memory, 3, 463-495.

Hodges, J. R., \& Patterson, K. (1995). Is semantic memory consistently impaired early in the course of Alzheimer's disease? Neuroanatomical and diagnostic implications. Neuropsychologia, 33, 441-459.

Hodges, J. R., Patterson, K., Oxbury, S., \& Funnell, E. (1992). Semantic dementia: Progressive fluent aphasia with temporal lobe atrophy. Brain, 115, 1783-1806.

HuMPHREYs, G. W., \& Forde, E. M. (2001). Hierarchies, similarity, and interactivity in object recognition: On the multiplicity of "categoryspecific" deficits in neuropsychological populations. Behavioral \& Brain Sciences, 24, 453-509.

JOSEPH, J. E. (2001). Functional neuroimaging studies of category specificity in object recognition: A critical review and meta-analysis. Cognitive, Affective, \& Behavioral Neuroscience, 1, 119-136.

Lambon Ralph, M. A., McClelland, J. L., Patterson, K., GalTON, C. J., \& HodGES, J. R. (2001). No right to speak? The relationship between object naming and semantic impairment: Neuropsychological evidence and a computational model. Journal of Cognitive Neuroscience, 13, 341-356.

Levy, D. A., Bayley, P. J., \& Squire, L. R. (2004). The anatomy of semantic knowledge: Medial vs. lateral temporal lobe. Proceedings of the National Academy of Sciences, 101, 6710-6715.

Marinkovic, K., Dhond, R. P., Dale, A. M., Glessner, M., Carr, V., \& Halgren, E. (2003). Spatiotemporal dynamics of modalityspecific and supramodal word processing. Neuron, 38, 487-497.

Martin, A., \& ChaO, L. L. (2001). Semantic memory in the brain: Structure and processes. Current Opinion in Neurobiology, 11, 194-201.

Mazoyer, B. M., Tzourio, N., Frak, V., Syrota, A., Murayama, N., LEVRIER, O., ET AL. (1993). The cortical representation of speech. Journal of Cognitive Neuroscience, 5, 467-479.

McClelland, J. L., \& Rogers, T. T. (2003). The parallel distributed processing approach to semantic cognition. Nature Reviews Neuroscience, 4, 310-322.

Moore, C., \& Price, C. (1999). A functional neuroimaging study of the variables that generate category-specific object processing differences. Brain, 122, 943-962.

Moss, H. E., Rodd, J. M., Stamatakis, E. A., Bright, P., \& Tyler, L. K. (2005). Anteromedial temporal cortex supports fine grained differentiation among objects. Cerebral Cortex, 15, 626-627.

Mummery, C. J., Patterson, K., Price, C. J., Ashburner, J., FrackOWIAK, R. S. J., \& HODGES, J. (2000). A voxel-based morphometry study of semantic dementia: Relationship between temporal lobe atrophy and semantic memory. Annals of Neurology, 47, 36-45.

Mummery, C. J., Patterson, K., Wise, R. J. S., Vandenberghe, R., Price, C. J., \& Hodges, J. R. (1999). Disrupted temporal lobe connections in semantic dementia. Brain, 122, 61-73.

Nakamura, K., Kawashima, R., Sato, N., Nakamura, A., Sugiura, M., Kato, T., ET AL. (2000). Functional delineation of the human occipito-temporal areas related to faces and scene processing: A PET study. Brain \& Cognition, 123, 1903-1912.

Nakamura, K., Kawashima, R., Sugiura, M., Kato, T., Nakamura, A., HATANO, K., ET AL. (2001). Neural substrates for recognition of familiar voices: A PET study. Neuropsychologia, 39, 1047-1054.

Papagno, C., \& Capitani, E. (2001). Slowly progressive aphasia: A four-year follow-up study. Neuropsychologia, 39, 678-686.

Patterson, K., \& Hodges, J. (2000). Semantic dementia: One window on the structure and organisation of semantic memory. In J. Cermak (Ed.), Handbook of neuropsychology: Vol. 2. Memory and its disorders (pp. 313-333). Amsterdam: Elsevier.

Patterson, K., Lambon Ralph, M. A., Jeffries, E. A., Woollams, A., Jones, R., Hodges, J. R., \& Rogers, T. T. (2006). “Presemantic” cognition in semantic dementia: Six deficits in search of an explanation. Journal of Cognitive Neuroscience, 18, 169-183.

Perani, D., Cappa, S. F., Bettinardi, V., Bressi, S., Gorno-Tempini, M., Matarrese, M., \& Fazio, F. (1995). Different neural systems for the recognition of animals and man-made tools. NeuroReport, 6, 16371641 .
Plaut, D. C., \& Shallice, T. (1993). Deep dyslexia: A case study of connectionist neuropsychology. Cognitive Neuropsychology, 10, 377-500.

Ricci, P. T., Zelkowicz, B. J., Nebes, R. D., Meltzer, C. C., MinTUN, M. A., \& BECKER, J. T. (1999). Functional neuroanatomy of semantic memory: Recognition of semantic associations. Neurolmage, 9, 88-96.

Rogers, T. T., Hocking, J., Mechelli, A., Patterson, K., \& Price, C. (2005). Fusiform activation to animals is driven by the process, not the stimulus. Journal of Cognitive Neuroscience, 17, 434-445.

Rogers, T. T., Lambon Ralph, M. A., Garrard, P., Bozeat, S., McClelland, J. L., Hodges, J. R., \& Patterson, K. (2004). The structure and deterioration of semantic memory: A computational and neuropsychological investigation. Psychological Review, 111, 205-235.

Rogers, T. T., Lambon Ralph, M. A., Hodges, J. R., \& Patterson, K. (2003). Object recognition under semantic impairment: The effects of conceptual regularities on perceptual decisions. Language \& Cognitive Processes, 18, 625-662.

Rogers, T. T., \& McClelland, J. L. (2004). Semantic cognition: A parallel distributed processing approach. Cambridge, MA: MIT Press.

Rogers, T. T., Watling, L., Hodges, J. R., \& Patterson, K. (2005, April). A basic-level disadvantage for speeded category verification. Paper presented at the meeting of the Cognitive Neuroscience Society, New York.

Rosch, E., Mervis, C. B., Gray, W., Johnson, D., \& Boyes-Braem, P. (1976). Basic objects in natural categories. Cognitive Psychology, 8 , 382-439.

Scott, S., Blank, C. C., Rosen, S., \& Wise, R. (2000). Identification of a pathway for intelligible speech in the left temporal lobe. Brain, 124, 83-95.

Scott, S., LEFF, A., \& Wise, R. J. (2003). Going beyond the information given: A neural system supporting semantic interpretation. NeuroImage, 19, 870-876.

Sharp, D. J., Scott, S., \& Wise, R. J. (2004). Monitoring and the controlled processing of meaning: Distinct prefrontal systems. Cerebral Cortex, 14, 1-10.

SnOdgrass, J. G., \& VANDERWART, M. (1980). A standardized set of 260 pictures: Norms for name agreement, image agreement, familiarity, and visual complexity. Journal of Experimental Psychology: Learning, Memory, \& Cognition, 6, 174-215.

Snowden, J. S., Goulding, P. J., \& Neary, D. (1989). Semantic dementia: A form of circumscribed temporal atrophy. Behavioural Neurology, 2, 167-182.

Snowden, J. S., Neary, D., \& Mann, D. M. A. (1996). Frontotemporal lobar degeneration: Frontotemporal dementia, progressive aphasia, semantic dementia. New York: Churchill Livingstone.

Sugiura, M., Kawashima, R., NaKamura, K., Sato, N., Nakamura, A., KATO, T., ET AL. (2001). Activation reduction in anterior temporal cortices during repeated recognition of faces of personal acquaintances. NeuroImage, 13, 877-890.

THOMPSON-SCHILL, S. L. (2003). Neuroimaging studies of semantic memory: Inferring "how" from "where." Neuropsychologia, 41, 280-292.

Thompson-Schill, S. L., Aguirre, G. K., D’Esposito, M., \& Farah, M. J. (1999). A neural basis for category and modality specificity of semantic knowledge. Neuropsychologia, 37, 671-676.

Tranel, D., Damasio, H., \& Damasio, A. R. (1997). A neural basis for the retrieval of conceptual knowledge. Neuropsychologia, 35, 13191327.

Tyler, L. K., Stamatakis, E. A., Bright, P., Acres, K., Abdallah, S., RoDd, J. M., \& Moss, H. E. (2004). Processing objects at different levels of specificity. Journal of Cognitive Neuroscience, 16, 351-362.

Vandenberghe, R., Nobre, A. C., \& Price, C. J. (2002). The response of left temporal cortex to sentences. Journal of Cognitive Neuroscience, 14, 550-560.

Vandenberghe, R., Price, C., Wise, R., Josephs, O., \& FrackoWIAK, R. S. J. (1996). Functional anatomy of a common semantic system for words and pictures. Nature, 383, 254-255.

WARRINGTON, E. K. (1975). Selective impairment of semantic memory. Quarterly Journal of Experimental Psychology, 27, 635-657.

(Manuscript received July 27, 2005; revision accepted for publication February 18, 2006.) 